\title{
Obstructive sleep apnoea as a risk factor for osteopenia and osteoporosis in the male population: further data and comments
}

To the Editor:

We have recently read some criticisms raised from SForZA et al. [1] in Sleep Medicine about our paper entitled "Obstructive sleep apnoea as a risk factor for osteopenia and osteoporosis in the male population" published in the European Respiratory Journal last March [2]. In the latter, we reported the significant reduction of the bone mineral density in a large population of male severe obstructive sleep apnoea (OSA) patients (apnoea-hypopnoea index $>30$ events per h) compared to a population of age- and body mass index (BMI)-matched controls [2]. Since we published our report as a research letter we could not provide the full data, neither in the manuscript nor in the form of supplementary material [2]. For this reason we write this correspondence in order to specify our results and to respond to the criticisms from SForzA et al. [1], and thank the Editor for giving us this opportunity. We would specify that in the previous study of our group we included consecutive male OSA patients admitted to our outpatient clinic, who underwent polygraphic cardiorespiratory monitoring according to the American Academy of Sleep Medicine criteria [3]. Since the aim of our study was to evaluate the possible influence of severe OSA on bone mineral density in the male population, we ruled out possible confounding factors that might have reduced the reliability of our results by excluding all the patients who presented one or more conditions with known influence on bone health (see exclusion criteria of the previous paper) [2]. Therefore, we excluded 148 out of 240 male OSA patients since: 67 were affected by diabetes, 31 had chronic obstructive pulmonary disease, four showed thyroid dysfunction, three reported use of corticosteroids in the 4 weeks preceding the inclusion in the study, 29 reported diuretic treatments, nine reported vitamin supplements consumption and five had history of fractures. Moreover, we compared the group of 92 male OSA patients to a group of 50 male subjects who were age- and BMI-matched with OSA patients not showing OSA syndrome (OSAS) at the polygraphic recording. OSAS has been defined according to the recent International Classification of Sleep Disorder, 3rd edition (ICSD3) (table 1) [4].

We selectively conducted the study in men since osteoporosis is a growing health problem in the male population, which needs of a more complete picture of prevalence and aetiopathogenesis of this disabling disorder. Moreover, this report followed a previous observation from our group about vitamin D deficiency in the OSA population [5]. In particular, we showed that vitamin D homeostasis is severely impaired in severe OSA patients, but could be positively modified by positive airway pressure treatment in the male population [5]. Therefore, we did not include women in the following studies about the long term effect of positive airway pressure treatment on vitamin D status in OSA patients and about the effects of OSA on bone mineral density $[2,6]$. We based our study designs in keeping with the following observations: 1) women did not ameliorate their vitamin D status after positive airway pressure therapy [5]; 2) osteoporosis is more frequent in women, who frequently show changes in sexual hormones due to postmenopausal condition, which primary influences bone turnover and metabolism [7]; 3) owing to the consecutive enrolment of the study protocol and considering that OSA is more prevalent in men, we excluded women given that the statistical comparison between female and male groups could be clearly difficult to obtain [8]; and 4) since we included young to elderly patients, women showing significantly different sexual hormones states due to the occurrence of menopause do not constitute an homogeneous

@ERSpublications

Obstructive sleep apnoea as a risk factor for osteopenia and osteoporosis in males: further data and comments http://ow.ly/RNCJ309KtPp

Cite this article as: Liguori C, Piccirilli E, Izzi F, et al. Obstructive sleep apnoea as a risk factor for osteopenia and osteoporosis in the male population: further data and comments. Eur Respir J 2017; 49: 1602471 [https://doi.org/10.1183/13993003.02471-2016]. 


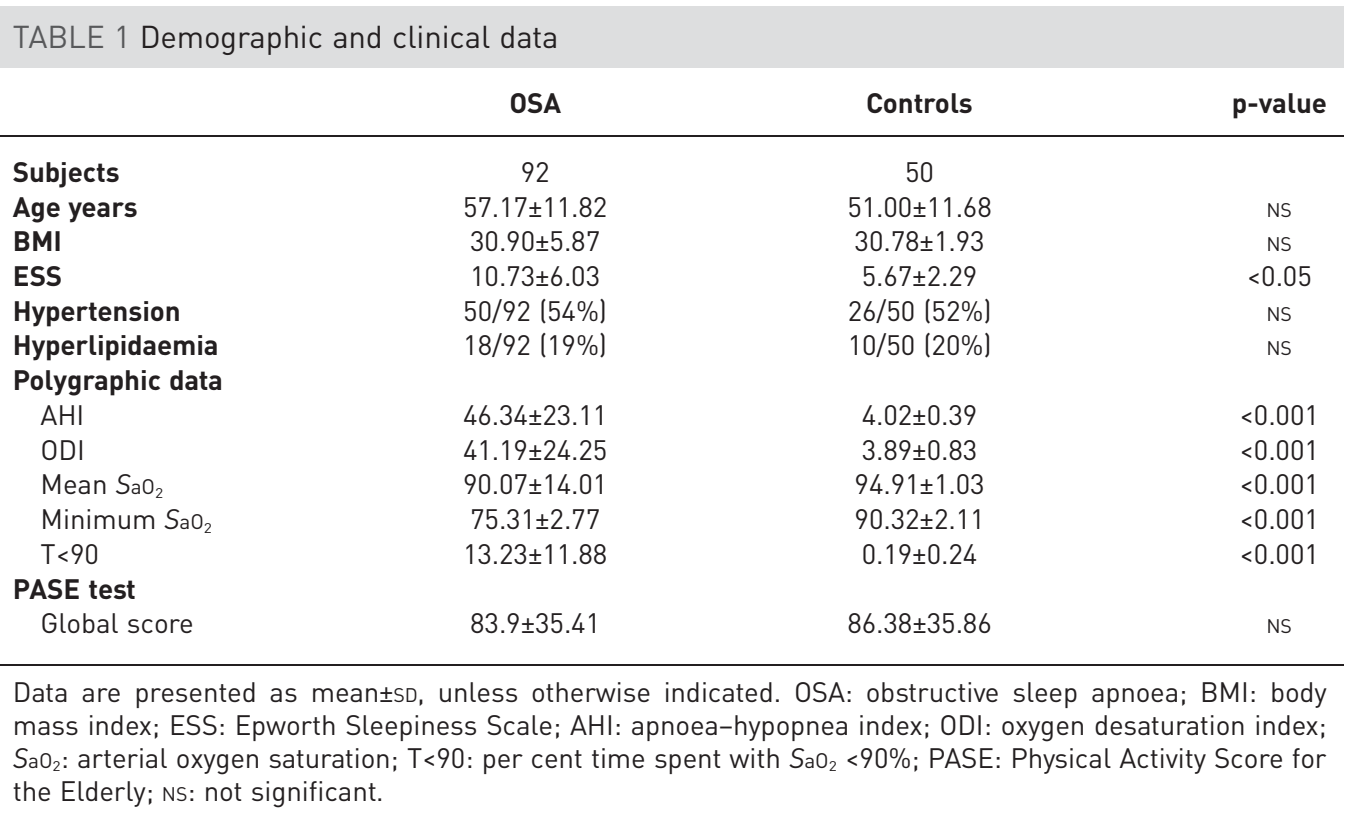

population [7]. Indeed, considering that the previous observation on vitamin D status in female patients was performed in a population of young to elderly subjects, and possibly counting pre-, peri- and post-menopausal women that could additionally give a bias to our analysis, we specifically include men in the following studies $[2,5,6]$.

Considering the complexity of the study consisting of the recruitment of a large group of young to elderly subjects in an outpatient setting, we chose a self-reported easy questionnaire to check our population of patients and controls for physical activity, such as the Physical Activity Score for the Elderly (PASE) test. We are aware that this scale is primarily focused on evaluating the physical activity of the elderly, however it has been already used in groups of patients counting adult to elderly subjects [9]. Moreover, it has been previously validated as an Italian version [10].

In conclusion, although a more complete picture of the influence of OSA diagnosis on the bone mineral density is needed, we think that the paper from our group coupled with the reports from SForza et al. [1] could add significant evidence for the need of osteoporosis and fracture risks evaluation in OSA patients. Accordingly, a recently published report documented that poor sleep quality is associated with the lower bone stiffness index [11]. In this study, the reduced bone stiffness index, an expression of the alteration of bone health, is related to higher scores on the Pittsburgh Sleep Quality Index questionnaire and, in particular, to the item 5 describing the presence of sleep disturbances [11]. Hence, we invite future researches on the interplay among sleep, OSA and bone mineral density in the male and female populations at all ages.

Claudio Liguori ${ }^{1}$, Eleonora Piccirilli ${ }^{2}$, Francesca Izzi $^{1}$, Nicola Biagio Mercuri ${ }^{1,3,4}$, Umberto Tarantino ${ }^{2}$ and Fabio Placidi ${ }^{1}$

${ }^{1}$ Sleep Medicine Centre, Neurophysiopathology Unit, Dept of Systems Medicine, University of Rome "Tor Vergata", Rome, Italy. ${ }^{2}$ Dept of Orthopedics and Traumatology, University of Rome "Tor Vergata", Rome, Italy. ${ }^{3}$ Fondazione Santa Lucia IRCCS, Rome, Italy. ${ }^{4}$ Neurology Unit, Dept of Systems Medicine, University of Rome "Tor Vergata", Rome, Italy.

Correspondence: Claudio Liguori, Sleep Medicine Centre, Neurophysiopathology Unit, Department of Systems Medicine, University of Rome “Tor Vergata”, Viale Oxford 8100133 Rome, Italy. E-mail: dott.claudioliguori@yahoo.it

Received: Dec 162016 | Accepted: Jan 102017

Conflict of interest: None declared.

\section{References}

1 Sforza E, Saint Martin M, Thomas T, et al. Reply to "Letter to the editor: Physical activity differently affects bone mineral density in obstructive sleep apnea at different age". Sleep Med 2017; in press [https://doi.org/10.1016/j. sleep.2016.11.005].

2 Liguori C, Mercuri NB, Izzi F, et al. Obstructive sleep apnea as a risk factor for osteopenia and osteoporosis in the male population. Eur Respir J 2016; 47: 987-990.

3 Iber C, Ancoli-Israel S, Chesson AL Jr, et al. The AASM manual for the scoring of sleep and associated events: rules, terminology and technical specifications. 1st Edn. Westchester, American Academy of Sleep Medicine, 2007. 
International Classification of Sleep Disorders. 3rd Edn. Westchester, American Academy of Sleep Medicine, 2014. Liguori C, Romigi A, Izzi F, et al. Continuous positive airway pressure treatment increases serum vitamin D levels in male patients with obstructive sleep apnea. J Clin Sleep Med 2015; 11: 603-607.

6 Liguori C, Izzi F, Mercuri NB, et al. Vitamin D status of male OSAS patients improved after long-term CPAP treatment mainly in obese subjects. Sleep Med 2017; 29: 81-85.

7 Nguyen HT, von Schoultz B, Nguyen TV, et al. Sex hormone levels as determinants of bone mineral density and osteoporosis in Vietnamese women and men. J Bone Miner Metab 2015; 33: 658-665.

8 Heinzer R, Vat S, Marques-Vidal P, et al. Prevalence of sleep-disordered breathing in the general population: the HypnoLaus study. Lancet Respir Med 2015; 3: 310-318.

9 Szabo AN, McAuley E, Erickson KI, et al. Cardiorespiratory fitness, hippocampal volume, and frequency of forgetting in older adults. Neuropsychology 2011; 25: 545-553.

10 Abete P, Ferrara N, Cacciatore F, et al. High level of physical activity preserves the cardioprotective effect of preinfarction angina in elderly patients. J Am Coll Cardiol 2001; 38: 1357-1365.

11 Sasaki N, Fujiwara S, Yamashita H, et al. Impact of sleep on osteoporosis: sleep quality is associated with bone stiffness index. Sleep Med 2016; 25: 73-77. 